\title{
Underwater Wireless Sensor Networks
}

\author{
S.Sweta ${ }^{\#}$, Balajee Maram ${ }^{\#}$ \\ " CSE, GMRIT, Rajam Andhra Pradesh, India \\ E-mail : suravajhalasweta123@gmail.com,balajee.m@gmrit.org
}

\begin{abstract}
There are a plenty of unexploited resources that lies underwater that covers almost $75 \%$ of the earth.In order to utilise them,the field of underwater wireless sensor networks (UWSN) is attracting the researchers to extend their thoughts in this field. The wireless sensor networks are heavy networks that consist of small low cost sensors that have a large amount of solving ability and energy resources which can be applicable in any type of irregular environments irrespective of changing conditions. Keeping in view of the real-time remote data transferring requirements, underwater acoustic sensor networks (UASN) has been recognised as a preferred network because it satisfies all aspects of data transfer. In UASN, the required availability and recycling of energy resources along with specified utilisation of data with the help of utilized sensor nodes for energy requirements that are necessary are done for the development of further theories in these contexts. Due to these causes, the maximum underwater resources utilisation techniques mainly depends on UAN (Underwater Acoustic Networks).Underwater wireless sensor networks (UWSNs) suitable for applications on submarine detection and monitoring,where nodes collect data with a mobile autonomous underwater vehicle (AUV) via optical communications, and applied accordingly to deal with further approaches. They provide continuous monitoring for various applications like ocean sampling network, pollution monitoring, submarine detection, disaster prevention etc.This paper particularly deals with a brief collection of the UWSN applications and some of the algorithms for the path finding in order to pass maximum valued information(VOI) among the different nodes.
\end{abstract}

Keywords - Underwater wireless sensor networks (UWSN), underwater acoustic networks (UAN), Autonomous underwater vehicle (AUV), Value of Information (VOI).

\section{INTRODUCTION}

The $3 / 4$ Th part of earth is covered by water and it implies that some of the underwater explorations should be done. We know a little about our oceans and what lies under it. This may contribute to many applications which are still hidden underwater and are related to a large variety of military and non-military applications like agriculture, coastal and protections, telecommunications, and climate controls and detection measures, search missions etc. Hence underwater sensor networks has a burning issue in the growing field oceanic research mainly. The research has been explained by the comparison between different types of underwater sensor networks. In the underwater environment, the channel is very difficult as compared to other networks in nature and is also related to altitude factors. The medium may be varying i.e. sometimes monstrous and sometimes saline in nature as underwater components. So, in order to deal with all the underwater suitable characteristics a technic is needed that should be suitable to each and every nature without any interruptions. As compared to other introduced networks like terrestrial and radio frequency, acoustic waves have a less underwater conduction velocity, the sensor nodes are bigger in size and the energy resources are very rarely found which cannot be recycled and reused. So, everything depends on energy consumption, the percentage of genuine utilization and also on the factor of long distance connectivity. As mentioned before an external mobile autonomous device is mainly used for routing the transferred data from one place to another efficiently and are cheap as compared to other communications.

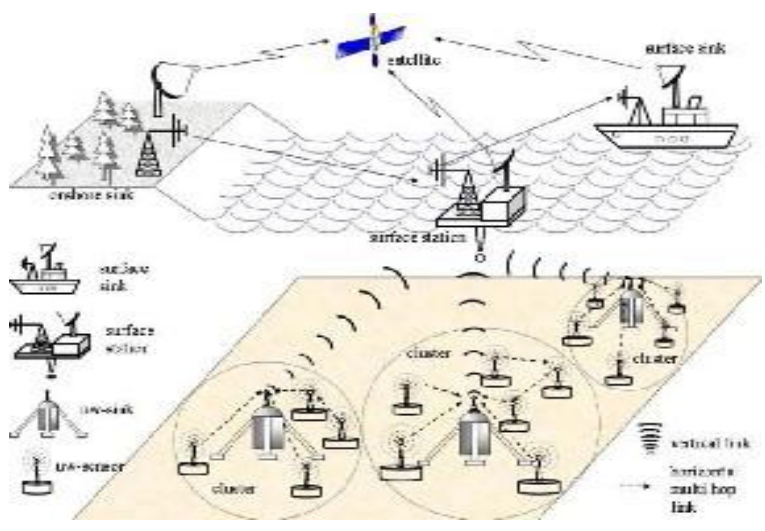

Fig 1. Underwater view when devices are work 
Transferring of large amounts of data in any communication is difficult if it does not have a shortest path among them. So, while transferring large and high-quality data like video, sampled data it may create a less efficiency using these networks, but unlike other communications they are strong so they are mostly preferred for short distance communications. The sensor nodes are the main components. The sensor nodes sense and record data and are linked with the AUV which in turn collects those gathered data and keeps them settled. However, this process should be done smartly such that it should not affect the other parameters. Nodes consists of acoustic modems to exchange the transferred information with AUVs. Data produced by a node sensing an event varies in size, value and the time in which it has to be delivered as compared to other introduced networks like terrestrial and radio frequency.

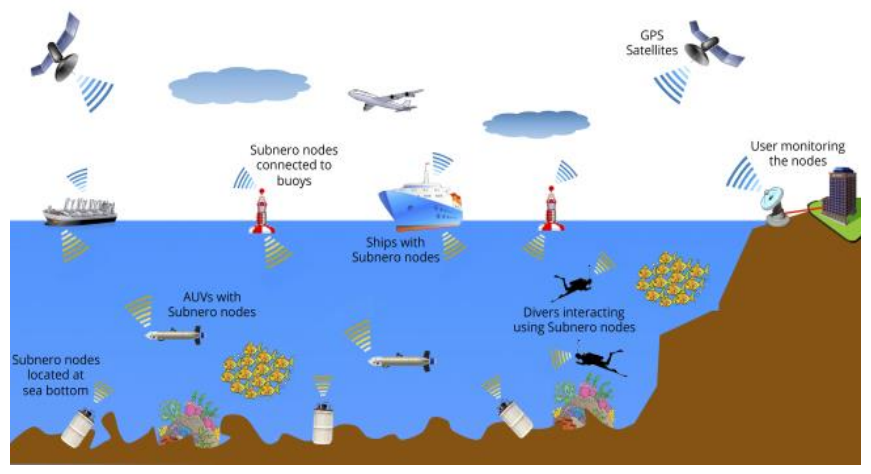

Fig 2.underwater view of the AUV visiting nodes

1. A new Integer Linear Programming model for finding AUV paths that maximizes the VOI of data to be transmitted to the destination. This provides certain boundaries on the basis of optimal strategies for protocols. Whatever the strategy may be it does not have any restrictions. It just does its work. This method also focuses on other parameters like transmission rate, speed of detection, speed of receival etc.which are the most important factors while transferring data among two stations. This was the first method introduced in account of VoI.

2. Another matter regarding this is the GAAP (Greedy and Adaptive AUV Path finding). This is implemented by using evaluation of performance in UWSN where the main components are the nodes, time taken, the time taken for complete replenish. This method produces $80 \%$ of the VoI on the basis of results. Compared to the other related techniques it is capable of producing 50$70 \%$ of the efficient information at the sink.

These all techniques are used for detecting and analysis of oceanic and underwater resources. Some of the design issues and the architecture of the different devices used are explained in the next sections.

\section{GREEDY HEURISTIC}

As mentioned above this is the latest technique used in underwater wireless sensor networks.But there is a certain procedure that includes the algorithms which takes some inputs, works on it and gives result. So in order to implement this method some of the algorithms are to be performed.The main principle is that data exists everywhere but the way the things are gathered is most important. Here in this methodology a new device called AUV (Acoustic Underwater Vehicle) has been introduced which is capable of collecting the data by travelling point to point. After collecting data from each node it arrives at a particular location to deliver them and finally after this process has done with each and every location, it comes back to the initial location. This procedure continues for all the nodes. When the data is gathered then as soon as possible an data frame is created depending upon the certain value of existing specified and other parameters. The data frame or packet taken is transmitted to the AUV using any mode of communication. The node that has given information to the AUV starts keeping track of the updated information and takes a value called $\mathrm{v}$ which describes the velocity at which it travels and the time interval i.e. at each unit the production of packets.

The AUV follows the greedy strategy i.e. collecting data from each and every small unit and then combining the whole data so as to obtain a complete big unit. Since it follows the Greedy Approach so this method is named as Greedy and Adaptive Path Finding method. It contributes the maximum amount of VoI by visiting the nodes. The main component of this algorithm is production of $\mathrm{VoI}$ by visiting a sensor node Si.The algorithm takes input as the data collected by each node and then works on it. The node ID Si the time taken to travel and produce a data frame as v. The other parameters are total time, time taken for receivable etc. The algorithm is given as follows:

\section{Algorithm 1: VoIFromNode(Si ,v , VoI info, T $i, t c, T)$}

The AUV follows a greedy strategy for visiting the nodes.

The algorithm takes input as the data collected by each node and then works on it. The node ID Si the time taken to travel and produce a data frame as v.The other parameters are total time taken for receivable etc.Thus it helps in finding an optimal solution. The algorithm is specified as the following:

Si: Strategy of collection and delivery

Ld= VoI-based queue of data chunks info;

VTiSi=0;

$t f i=0$;

for $\Psi 1$ to $|L d| d o$

$V o I^{\prime}=\sum$ VoI of data chunks delivered ' $\Psi$ at a time; $6 t \Psi$ = time it takes to collect and deliver all data

if VoI $\Psi$ VTiSi then

$t f i=t c+t \Psi$

if $t f i>T$ then break;

VTisi $=V o I \Psi$

$S i=$ Deliver $\Psi$ data chunks at a time;

return (VTisi, tfi , Si); 


\section{Algorithm 2: Node Selection(VoI Info)}

This algorithm mainly concentrates on the node selection, before performing the VoI method. Atfirst the nodes has to be selected in order to continue the process. They are indulged in above algorithms. One of the main perspective is that each of the node.

$$
\begin{aligned}
& \text { Node Sk to be visited } \\
& E=\text { Set of nodes sensing an event; } \\
& S i=\text { Node in } E \text { with the highest } V t i \\
& (\text { Sk }, \text { scorek }=(0,0) \text {; } \\
& \text { for } S j \text { ć } E: S j \neq S i \text { do } \\
& (\text { Si } \mid j, \text { scorei } \mid j i=\text { CombinedVoI-Score }(S i, T i, S j, T j) \text {; } \\
& \text { if scorei|j > scorek then } \\
& (S k, \text { scorek })=(\text { Si } \mid j, \text { scorei } \mid j) \text { return } S k \text {; }
\end{aligned}
$$

\section{LITERATURE SURVEY}

Petrica gjanci, Chiara petrioli, Stefano Basagni, Cynthia A. Philips, Ladisau boloni and Damla Turgut, IEEE 2706689,2017 . In this paper[1] we presented a mathematical model(OPT) a greedy heuristic (GAAP) for driving an AUV to collect and deliver data with decaying Value of Information of the data delivered to that sink. Our ILP communication rates, distances and surfacing constraints.

D N Sandeep and Vinay Kumar proposed a paper [2] on "Review on clustering, coverage and connectivity in underwater wireless sensor networks, communication techniques perspective". This paper deals with the latest underwater acoustic clustering techniques and a comparative analysis is done with respect to various performance parameters. Various schemes by which the coverage can be maximised and simultaneously providing the value of information by adjusting the depth of nodes or by using autonomous underwater vehicles are summarized.

IEEE Farr N. Bowen ,A. Ware, J., Pontbriand, C. \& Tivey, M.2010 "An integrated, underwater, optical / acoustic communications system. This paper [3] deals with the underwater acoustic sensor networks which are budding and following the path of radio frequency in different networks.An overview of the state of the art in underwater channels. The ultimate objective is to encourage and specify the importance of research efforts to lay down new advanced technology communication rates, distances and surfacing constraints.

\section{CONCLUSIONS}

Since there are several methods for AUV path finding but the GAAP or greedy heuristic, the main objective is mainly to find paths for the AUV to take the data and transfer maximum value of information at the final end. The GAAP perfectly provides the best routing paths formed by all other algorithms and finally gives VoI of whatever the data delivered by the vehicle which is almost $30 \%$ better than others. The performance of GAAP as compared to other path finding techniques provides $75 \%$ of the more VoI because it continuously visits nodes and also satisfies the overall energy efficiency that is also $70 \%$ better than that of other methods.So GAAP should be preferred more in the underwater wireless networks.

\section{REFERENCES}

[1] Petrica gjanci ,Chiara petrioli, Stefano Basagni ,Cynthia A.Philips,Ladisau boloni and Damla Turgut,IEEE 2706689,2017.

[2] D N Sandeep and Vinay Kumar proposed 2015, "Review on clustering, coverage and connectivity in underwater wireless sensor networks, communication techniques perspective"

[3] IEEE Farr N. Bowen ,A.Ware, J., Pontbriand, C.\&Tivey, M.2010 "An integrated ,underwater,optical/acoustic communications system.

[4] Ji Li, Lachlan LH Andrew, Chuan Heng Foh, Moshe Zukerman, and Hsiao- Hwa Chen. Connectivity, coverage and placement in wireless sensor networks. Sensors, 9(10):7664-7693, 2009

[5] P. A. Forero, S. K. Lapic, C. Wakayama, and M. Zorzi. Rollout algorithms for data storage-and energy-aware data retrieval using autonomous underwater vehicles. In Proceedings of ACM WUWNet 2014, pages 22:1-22:8, Rome, Italy, November 12-14 2014.

[6] C. Bisdikian, J. Branch, K. K. Leung, and R. I. Young. A letter soup for the quality of information in sensor networks. In Proceedings of IQ2S 2009, pages 1-6, Galveston, TX, March 92009.

[7] G. A. Hollinger, S. Choudhary, P. Qarabaqi, C. Murphy, U. Mitra, G. S. Sukhatme, M. Stojanovic, H. Singh, and F. Hover. Underwater data collection using robotic sensor networks. IEEE Journal on Selected Areas in Communications, 30(5):899-911, 2012.

[8] D. Turgut and L. Bölöni. IVE: Improving the value of information in energy-constrained intruder tracking sensor networks. In Proceedings of IEEE ICC 2013, pages 4953-4957, Budapest, Hungary, June9132013C. Bisdikian, L. M. Kaplan, M. B. Srivastava, D. J. Thornley, D. Verma, and R. I. Young. Building principles for a quality of information specification for sensor information. In Proceedings of IEEEFUSION2009, pages1370-1377, Seattle, WA,July6-9 2009.

[9] G. A. Hollinger, S. Choudhary, P. Qarabaqi, C. Murphy, U. Mitra, G. S. Sukhumi, M. Stojanovic, H. Singh, and F. Hover. Underwater data collection using robotic sensor networks. IEEE Journal on Selected Areas in Communications, 30(5):899-911, 2012.

[10] D. Turgut and L. Bölöni. A pragmatic value of information approach for intruder tracking sensor networks. In Proceedings of IEEEICC2012, pages4931-4936, Ottawa, Canada, June10-15 2012.

[11] D. Turgut and L. Bölöni. IVE: Improving the value of information in energy-constrained intruder tracking sensor networks. In Proceedings of IEEE ICC 2013, pages 4953-4957, Budapest, Hungary, June9132013.

[12] N. Farr, J. Ware, C. Pontbriand, T. Hammar, and M. Tivey. Optical communication system expands CORK seafloor observatory's bandwidth. In Proceedings of the MTS/IEEEOCEANS 2010, pages1-6, Seattle, WA, September20-232010.

[13] M. Doniec, C. Detweiler, I. Vasilescu, and D. Rus. Using optical communication for remote underwater robot operation. In Proceedings of IEEE/RSJ Intelligent Robotsand Systems, IROS 2010, pages4017-4022, Taipei, Taiwan, October18-222010. 